\title{
Are Pictures Worth a Thousand Words: A Pilot Randomized Trial of Vis- ual Cues to Improve Speed of Completion and Comprehension of Three Surveys for Older Adults
}

\author{
${ }^{I}$ The University of Queensland, Australia \\ ${ }^{2}$ Princess Alexandra Hospital, Australia \\ ${ }^{3}$ Monash University, Australia \\ ${ }^{4}$ Southern Health, Australia
}

Steven McPhail ${ }^{1,2}$, Elaine Beller ${ }^{1,2}$ and Terry Haines ${ }^{*}, 3,4$

\begin{abstract}
Background and Aims: When conducting surveys with older adults, it is important that participants have clear comprehension of the questions being asked. It is also important that survey items can be administered efficiently. This paper investigates the effect of providing visual cues to aid participant understanding and speed of completion of three types of health surveys.

Methods: Design - Pilot randomized controlled trial $(\mathrm{n}=12)$ of participants assigned to a visual cues (intervention) or no visual cues (control) group with unblinded outcome assessment using time taken to complete survey and question comprehension as outcome measures.

Results: Although participants from both groups did not report difficulty in understanding or remembering each question, the visual cues group were able to complete the surveys in significantly less time and with significantly fewer errors in comprehension than the no cues group.

Conclusions: The results of this study indicate that visual cues improved speed of survey completion and patient comprehension of survey items. This investigation suggests the use of visual cues may enhance efficiency and reduce labor costs in survey related research with the elderly and potentially reduce burden on participants. Furthermore, this may result in higher validity of questionnaires due to improved comprehension. The findings of this pilot study were unexpectedly definitive on their own merit such that a larger investigation was deemed to be unwarranted for the purpose of addressing the research aims.
\end{abstract}

Keywords: Aged, comprehension, surveys, questionnaires, visual aids.

\section{INTRODUCTION}

Surveys are widely used amongst older adults for a range of health evaluation purposes in both clinical and research contexts. Patient completed and interviewer administered surveys and questionnaires have been used for measurement of complex phenomena such as self rated global health, health-related quality of life, fear of falling, fatigue and functional impairment [1-7]. When conducting surveys with older adults, it is important that participants have clear comprehension of the questions they are being asked. It is also important that survey items can be administered expeditiously to ensure that research resources consumed and patient time burden are not excessive during questionnaire completion.

Benefits of using visual cues as an adjunct in questionnaires and other written materials have been investigated [711], but their effect on speed of completion and

*Address correspondence to this author at the Allied Health Clinical Research Unit, Kingston Centre, Cnr Warrigal and Kingston Rds, Cheltenham, Victoria, Australia 3192; Tel: +61 3 92651774;

E-mail: terrence.haines@med.monash.edu.au comprehension of health surveys by older adults has not been investigated. This paper describes a pilot investigation of patient comprehension and speed of completion of three surveys, using the survey pretesting method of probing questions [12]. It was hypothesized that patients who received visual cues would complete the surveys in less time and with fewer errors in comprehension.

\section{MATERIALS AND METHODOLOGY}

\section{Design}

Cognitive pre-testing of three surveys using a randomized controlled trial research design. Participants were assigned to a visual cues (intervention) or no visual cues (control) group, with unblinded outcome assessment (Fig. 1).

\section{Participants and Setting}

Rehabilitation inpatients from the Geriatric Assessment and Rehabilitation Unit (GARU) of a major tertiary hospital in Brisbane, Australia, gave informed consent and were recruited. Participant inclusion criteria were (1) Patients recently transferred to the sub-acute rehabilitation wards of the hospital from any acute hospital ward; (2) age 65 and older; (3) basic cognitive functioning in tact indicated by a Mini 


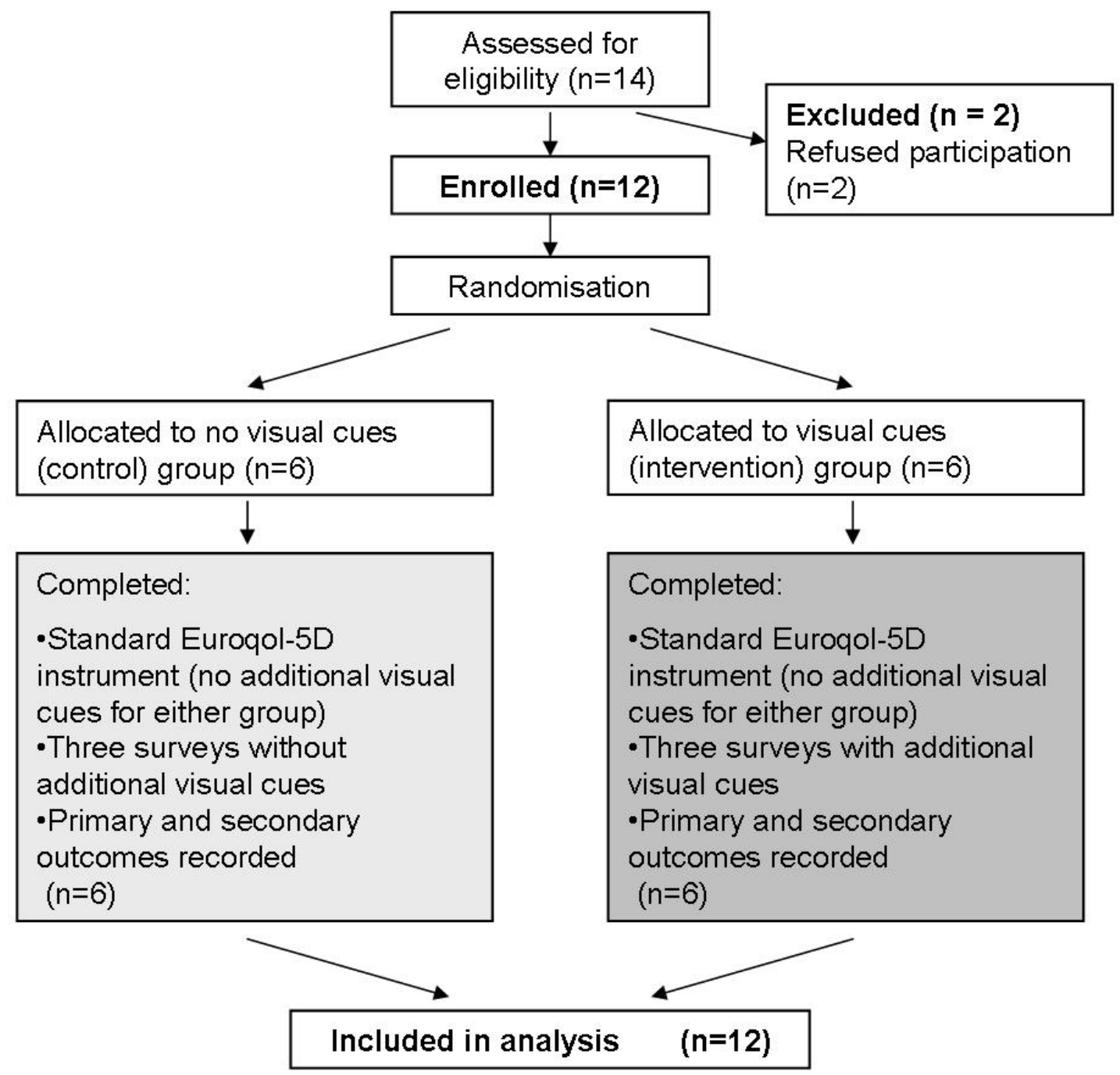

Fig. (1). Study design - randomised controlled trial.

Mental State Examination (MMSE) $>23 / 30$ [13]. The exclusion criteria was (1) an inability to communicate effectively in English (e.g. aphasic stroke patient). No formal sample size calculation was conducted due to the novelty and pilot nature of the investigation making estimates of effect size and standard deviation difficult. A sample size of twelve was initially selected with the intention of conducting analysis then either stopping this pilot investigation if a clear trend was evident, or using the information gathered to estimate a required sample size. Fourteen patients were identified as meeting the study criteria and approached for consent. One declined as she was about to leave the ward for the day, another declined as she was expecting visitors. Of the twelve consenting patients six were allocated to visual cues group and six were allocated to non-visual cues group. Eleven patients were from an English speaking background and the other had lived and worked in an English speaking country since childhood. No participants reported severe visual or auditory impairments; the use of usual eyewear such as reading glasses was permitted.

\section{Intervention}

Participants assigned to the visual cues group completed a survey interview by their bedside with the use of visual cues as an adjunct to the written text of the surveys under investigation. For participants assigned to the control group the same survey interview was administered without the visual cues for the surveys under investigation. Both groups of patients were given a copy of each question printed in large 22-point font before it was read aloud at equivalent pace for all participants by the interviewer.

The survey interview itself was a combination of the standard English version of the EuroQoL-5D (EQ-5D) [14] and three custom designed surveys investigating:

A. Patient knowledge of accidental falls Epidemiology (Epi)- Patients perceptions of actual inpatient falls and injury rates.

B. Health-Related Quality of Life (QoL)- Six imaginary falls scenarios, presented in random order for each patient, resulting in injuries and outcomes of varying severity. Participants were asked to report how they thought they would answer the EQ-5DVAS report at time periods of 4 weeks and 6 months post fall if they were in each scenario.

C. Willingness to Pay- (WTP) - Willingness to pay questions for six falls prevention strategies of equal 
effectiveness (a 30\% reduction in risk of falling), presented in random order, involving different levels of patient and ward staff participation. Participants were asked how much they would be willing to pay for the provision of various strategies during their current admission. Participants were advised that this one off payment would need to be made out of their own pocket at the time of discharge from hospital. Participants were also advised that these strategies were not currently available, but rather the purpose of the questions was to evaluate how highly hospital patients valued each of them.

Administration of the EQ-5D served as a control comparator condition between the two groups as no visual cues were provided for this survey regardless of group allocation.

The three remaining surveys were intended for use in subsequent investigations of these topics. The visual cues employed represented what was described with words in the scenarios or questions and were very simplistic in design (Fig. 2). Although the three surveys were somewhat related by genre, it is noteworthy that each survey type was different. The first (Epi) was a survey to investigate knowledge of a topic, the second (QoL) involved patients imagining themselves in complex scenarios and how that would effect their quality of life, and the last (WTP) was a willingness to pay questionnaire enquiring how much money the participant would be willing to sacrifice to attain a specific healthbenefit via the means specified in the question.

\section{Measures}

Participant baseline information including age, gender, diagnosis, prior living arrangements and cognitive status as measured by the Mini-Mental State Examination (MMSE) [13] were recorded. The primary outcome measure was time taken to complete each survey. Timing commenced once the participant had been provided with the question $(+/-$ visual cues) and ceased once an answer was provided. The secon- dary outcome measure was number of errors made when asked to recall comprehension of each question or scenario immediately after answering that question. Two participant self-report 5-point Likert scale scores were also recorded for each question, one asking whether patients found it easy to understand the question and one asking whether participants had difficulty remembering the entire question. This selfreport was recorded immediately after each question was answered.

\section{Procedure}

Potential participants were identified by their treating physiotherapist who sought verbal consent to be approached by one of the project investigators (SM). The project investigator then provided a verbal and written description of the project to the potential participant before seeking written informed consent. Participants were then randomized to receive either visual cues or no visual cues with the survey administration. A computer-generated random number sequence was developed by a project investigator (TH) not involved in participant recruitment or testing. The sequence was concealed in sequentially numbered opaque envelopes. The investigator undertaking recruitment and questionnaire administration assigned consecutive numbers to study participants in the order of recruitment and opened the same numbered envelope to reveal participant group allocation after participant demographic information had been collected. The EQ-5D instrument was then administered, followed by administration of the Epi, QoL, and WTP questionnaires.

\section{Analysis}

Mann-Whitney U tests were employed for planned comparisons to examine simple effects of group allocation for time to complete each individual survey. Completion times were also presented graphically by group and survey (Fig. 3). The total number of errors in comprehension and recall of key points in the three surveys were compared between

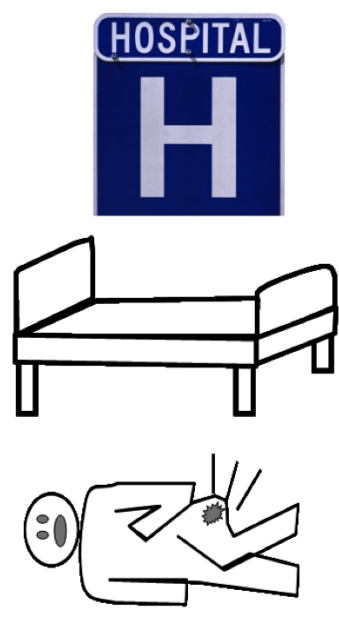

Now
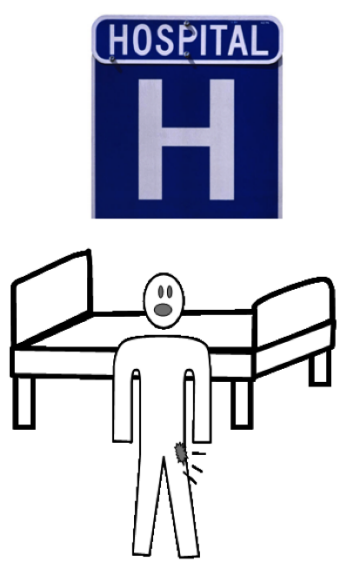

4 weeks

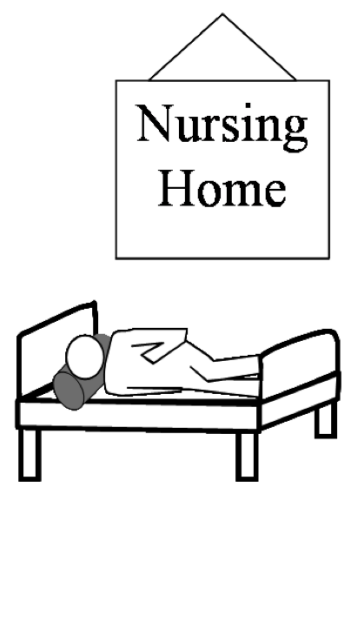

\section{6 months}

Fig. (2). An example of visual cues used for quality of life questions. 


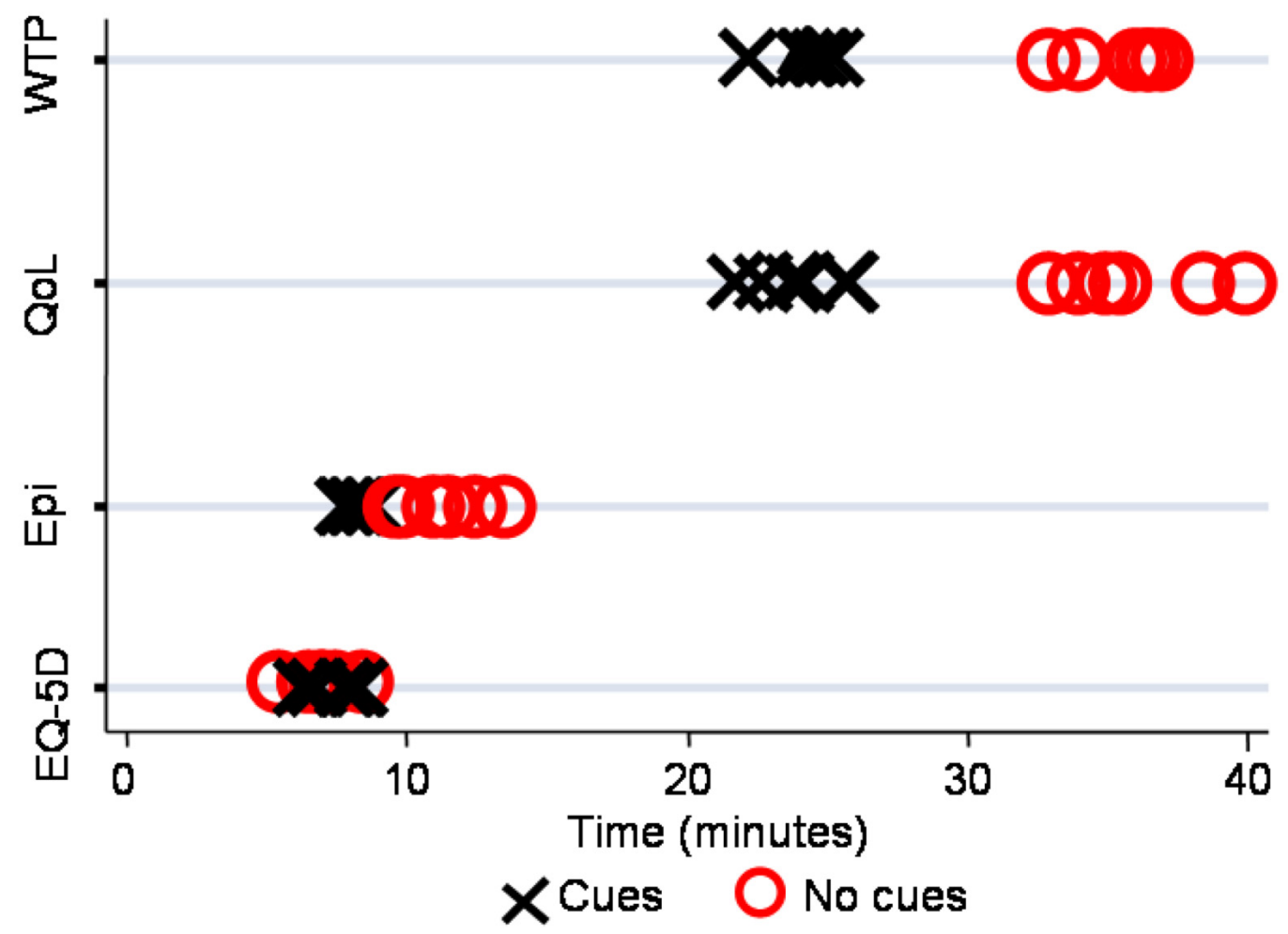

Fig. (3). Time taken to complete each survey, by group. WTP - Willingness to pay survey; QoL - Quality of life scenario survey; Epi Falls epidemiology survey; EQ-5D - Euroqol 5D questionnaire tool (English version - no visual cues for either group).

groups using negative binomial regression. The number of comprehension errors made for individual surveys by group were also presented in a frequency histogram (Fig. 4).

\section{Ethics}

This study was conducted in accordance with the ethical standards of the Princess Alexandra Hospital and The University of Queensland human research ethics committees.

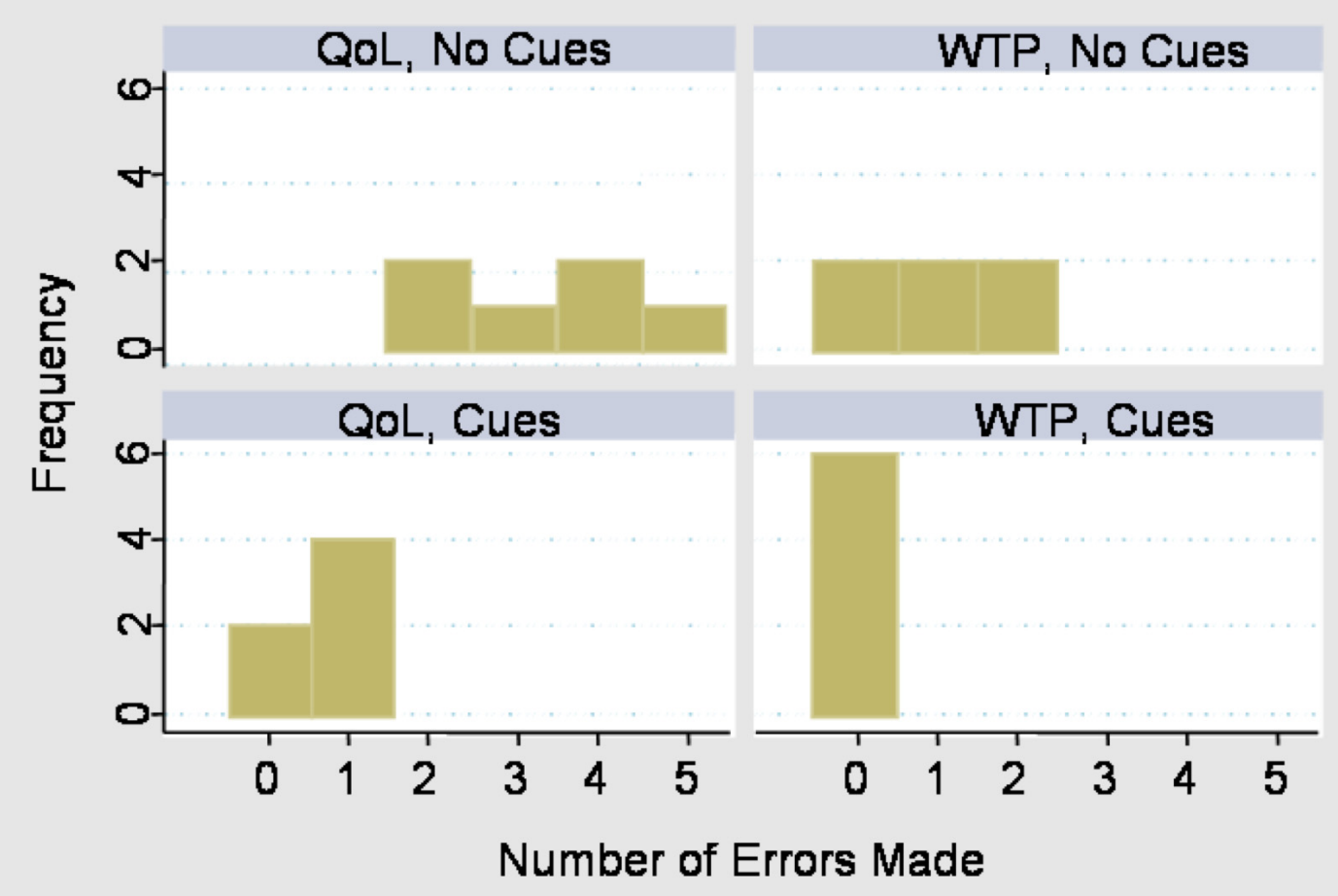

Fig. (4). Comprehension errors made by group and survey. QoL - Quality of life scenario survey; WTP - Willingness to pay survey. 
The study was conducted in January 2007.

\section{RESULTS}

All 12 participants completed the surveys without incident and all were included in the analysis. Baseline characteristics for both groups were similar and are displayed in Table 1. Participants admitted to this unit for rehabilitation due to age related impairments or disabilities such as muscular weakness or deconditioning due to illness were grouped under the "other geriatric management" diagnosis category.

Table 1. Participant Demographics

\begin{tabular}{|l|c|c|}
\hline \multicolumn{1}{|c|}{ Group } & $\begin{array}{c}\text { Intervention Group } \\
\text { (Visual Cues) }\end{array}$ & $\begin{array}{c}\text { Control Group } \\
\text { (No Cues) }\end{array}$ \\
\hline \hline $\mathrm{N}$ & 6 & 6 \\
\hline Mean age (range) & $75.5(65-85)$ & $75.3(65-83)$ \\
\hline $\begin{array}{l}\text { Gender (women) frequency } \\
(\%)\end{array}$ & $4(66 \%)$ & $5(83 \%)$ \\
\hline $\begin{array}{l}\text { Living in the community } \\
\text { frequency (\%) }\end{array}$ & $6(100 \%)$ & $6(100 \%)$ \\
\hline Mean MMSE (range) & $26.8(24-29)$ & $27.8(25-30)$ \\
\hline $\begin{array}{l}\text { Diagnosis frequency (\%) } \\
\text { Stroke }\end{array}$ & $1(17 \%)$ & $2(33 \%)$ \\
$\begin{array}{l}\text { Orthopedic } \\
\text { Other geriatric } \\
\text { management }\end{array}$ & $2(33 \%)$ & $3(50 \%)$ \\
& $3(50 \%)$ & $1(17 \%)$ \\
\hline
\end{tabular}

Significant simple effects of group were identified by the planned Mann-Whitney U tests for the three surveys where visual cues were provided for participants in the visual cues group, specifically the Epi $(\mathrm{p}=0.004)$, QoL $(\mathrm{p}=0.004)$ and WTP $(p=0.004)$, but not for the standardised EQ-5D survey $(p=0.570)$ which had no additional visual cues for either group. Representation of these simple effects can be seen in Fig. (3). The mean difference in time to complete the three surveys where visual cues were available was 27 minutes in total (Epi 3.25 minutes, QoL 12.25 minutes and WTP 11.5 minutes).

The number of errors in recalling key information made in each of the quality of life scenarios and willingness to pay questions are shown in Fig. (4). Errors made for the Epi survey are not presented as only one error was made by one participant in the no cues group. Overall, there were significantly fewer total errors made by the visual cues group [Incidence Rate Ratio $(95 \% \mathrm{CI})=0.11(0.03,0.37), \mathrm{p}<0.001]$.

Both groups had similar results for the patient self-report of understanding and level of difficulty remembering each question. No participants in either group agreed or strongly agreed that they had difficulty remembering the entire question or scenario in any part of the survey. In the same way no participants in either group reported they disagreed or strongly disagreed that any of the questions or scenarios in any part of the survey were easy to understand. In summary both groups gave similar self-reports of understanding and ease of remembering the entire question for each survey component despite the actual differences in comprehension.

\section{DISCUSSION}

The results of this study are the first to indicate that visual cues improve the speed of survey completion and patient comprehension. This result in itself suggests that the cognitive testing and pilot experimental use of visual cues for these surveys amongst older adults was successful and that visual cues should be used as an adjunct to the written text. This is the first study to evaluate an approach to facilitate participant understanding of a battery of survey items using a cognitive testing approach within a randomized controlled trial research design amongst older adults.

This investigation did not aim to investigate the underlying mechanism which caused the improved speed of completion and question comprehension; however several factors may have contributed. The visual cues may have helped generate interest in the survey questions and maintain concentration throughout survey completion. The visual cues may also have helped the older adults to understand and remember the multiple pieces of information presented during scenario and willingness to pay questions allowing them to respond promptly. This may also have improved their motivation and ability to attend to and subsequently comprehend each survey's questions.

The findings from this study have implications for others intending to use surveys to investigate complex constructs amongst elderly populations in both research and clinical practice. Specifically, the use of visual cues may enhance efficiency and reduce the amount of time that health professionals or research assistants spend conducting survey-based health evaluations and potentially reduce burden on patients / participants. Furthermore, this may result in higher validity of questionnaires due to improved comprehension. This study has also demonstrated survey instruments for older adults can be improved through the use of experimental and cognitive testing methods with minimal research resource usage.

The disparity between patient self report of understanding and self report of ease of remembering the entire question and the actual comprehension fits well with the elaborated model of question and answer processes previously reported when discussing cognitive aspects of survey methodology [12]. This model proposes non-linear interactions between comprehension, retrieval, judgment and response. In this process participants may choose to respond with the answer they want to give rather than the most correct answer. It is possible that participants in this study may not have wanted to report difficulty in understanding or remembering the survey questions. This further strengthens the argument for cognitive testing during pilot testing of survey instruments.

The extent to which the findings from the present study can be extrapolated more broadly are limited by several factors. The participants selected could be criticized as only being representative of a specific patient population, also the overall sample size was low. However, as significant differences were already identified through this pilot investigation, it was deemed of little additional value to pursue further recruitment to address the subject of this paper. Another consequence of a small sample size is an increased likelihood 
for differences between groups at baseline, particularly in key variables relating to the outcome measures of interest. However along with other demographic information, the two groups were equivalent in time to complete the standardised survey (EQ-5D) that did not contain visual cues for either group, indicating that their ability to complete surveys was most likely comparable at baseline. This study adds new, valuable information to the body of literature regarding research with older adults and indicates that the use of visual cues is likely to be beneficial to the speed of completion and level of comprehension during survey administration across various survey types.

Further research is warranted to investigate the value of visual cues for survey administration amongst other geriatric populations including those with a greater degree of cognitive impairment than those investigated in this study and those whose primary language is one other than that in which the survey is provided. Additionally, broader application of visual cues into the clinical setting, including patient education material, should be considered.

\section{CONCLUSIONS}

The results of this study indicate that the visual cues improve speed of survey completion and patient comprehension amongst hospitalized older adults. The use of visual cues may enhance efficiency and reduce labor costs in survey related research and in administration of survey based health assessments in clinical practice amongst older adults and potentially reduce burden on patients / participants. Furthermore, this may result in higher validity of questionnaires due to improved comprehension.

\section{Trial Registration:}

Australian New Zealand Clinical Trials Registry: ACTRN12606000097549: http://www.anzctr.org.au.

\section{REFERENCES}

[1] Gonzalez S, Huerta JM, Fernandez S, Patterson AM, Lasheras C. Life-quality indicators in elderly people are influenced by selenium status. Aging Clin Exp Res 2007; 19(1): 10-5.

[2] Vuorisalmi M, Lintonen T, Jylha M. Comparative vs global selfrated health: associations with age and functional ability. Aging Clin Exp Res 2006; 18(3): 211-7.

[3] Haywood KL, Garratt AM, Fitzpatrick R. Quality of life in older people: a structured review of generic self-assessed health instruments. Qual Life Res 2005; 14(7): 1651-68.

[4] Tinetti ME, Powell L. Fear of falling and low self-efficacy: a case of dependence in elderly persons. J Gerontol 1993; 48(Spec No): 35-8.

[5] Martinez-Martin P, Catalan MJ, Benito-Leon J, et al. Impact of fatigue in Parkinson's disease: The fatigue impact scale for daily use (D-FIS). Qual Life Res 2006; 15(4): 597-606.

[6] Wildner M, Sangha O, Clark DE, Doring A, Manstetten A. Independent living after fractures in the elderly. Osteoporos Int 2002; 13(7): 579-85.

[7] Coast J, Peters TJ, Richards SH, Gunnell DJ. Use of the EuroQoL among elderly acute care patients. Qual Life Res 1998; 7(1): 1-10.

[8] Larson CO, Hays RD, Nelson EC. Do the pictures influence scores on the Dartmouth COOP Charts? Qual Life Res 1992; 1(4): 247-9.

[9] Hadorn DC, Hays RD, Uebersax J, Hauber T. Improving task comprehension in the measurement of health state preferences. A trial of informational cartoon figures and a paired-comparison task. J Clin Epidemiol 1992; 45(3): 233-43.

[10] Kempen GI, van Sonderen E, Sanderman R. Measuring health status with the Dartmouth COOP charts in low-functioning elderly. Do the illustrations affect the outcomes? Qual Life Res 1997; 6(4): 323-8.

[11] Paul C, Redman S, Sanson-Fisher R. The development of a checklist of content and design characteristics for printed health education materials. Health Promot J Austr 1997; 7: 153-9.

[12] Collins D. Pretesting survey instruments: an overview of cognitive methods. Qual Life Res 2003; 12(3): 229-38.

[13] Folstein M, Folstein S, McHugh P. Mini-Mental State: a practical method for grading the cognitive state of patients for the clinician. J Psychiatr Res 1975; 12: 189-98.

[14] Rabin R, de Charro F. EQ-5D: a measure of health status from the EuroQol Group. Ann Med 2001; 33(5): 337-43.

(C) McPhail et al.; Licensee Bentham Open.

This is an open access article licensed under the terms of the Creative Commons Attribution Non-Commercial License (http://creativecommons.org/licenses/by$\mathrm{nc} / 3.0 /$ ) which permits unrestricted, non-commercial use, distribution and reproduction in any medium, provided the work is properly cited. 